OPEN ACCESS

Edited by:

Rita Covas,

University of Porto, Portugal

Reviewed by:

Kirsty MacLeod,

Lund University, Sweden

Per T. Smiseth

University of Edinburgh,

United Kingdom

${ }^{*}$ Correspondence:

James L. Savage

james.savage@cantab.net

Specialty section:

This article was submitted to Behavioral and Evolutionary Ecology,

a section of the journal

Frontiers in Ecology and Evolution

Received: 05 July 2019

Accepted: 18 October 2019

Published: 08 November 2019

Citation:

Savage $J L$ and Hinde CA (2019) What

Can We Quantify About Carer

Behavior? Front. Ecol. Evol. 7:418.

doi: 10.3389/fevo.2019.00418

\section{What Can We Quantify About Carer Behavior?}

\author{
James L. Savage ${ }^{1,2 \star}$ and Camilla A. Hinde ${ }^{3}$ \\ ${ }^{1}$ Department of Animal and Plant Sciences, University of Sheffield, Sheffield, United Kingdom, ${ }^{2}$ Department of Zoology, \\ University of Cambridge, Cambridge, United Kingdom, ${ }^{3}$ School of Life Sciences, Anglia Ruskin University, Cambridge, \\ United Kingdom
}

In many species, individuals must contribute extensively to offspring care to reproduce successfully. Within species, variation in care is driven by local social, physiological, and environmental contexts, and this relationship has been a major focus of behavioral ecology since the inception of the field. The majority of existing studies on care, both theoretical and empirical, have focused on measuring the amount of care delivered by each carer as a proxy for individual investment, linking this investment to the local context, and investigating outcomes for offspring. However, more recently interest has grown in the finer-scale details of care, including how individuals respond to each other's behavior, and temporal variation in care both within and between stages. Simultaneously, advances in remote monitoring methods, such as video cameras and passive integrated transponder (PIT) tag systems, have vastly increased the ease of collecting large amounts of care data, providing opportunities to study carer behavior in much greater detail than previously possible. In this mini-review we provide an overview of the dimensions of carer behavior that can be quantified, illustrated using recent studies from a variety of taxa. We classify these analyses into three broad groups: (a) how parental care is distributed in time, (b) variation within care events, and (c) how carers interact when jointly providing care. Our aim is to encourage more in-depth analyses of parental care, to build a more complete picture of how animals rear their offspring.

Keywords: cooperation, coordination, measuring behavior, parental care, provisioning, alternation, synchrony

\section{INTRODUCTION}

Parental care often requires substantial investment of time and energy, and strongly impacts the fitness of the individual carers that provide it (Saether, 1994). Previous studies have shown that care behavior is influenced by individual characteristics such as sex (Liker et al., 2015), age (Ortega et al., 2017), condition (Dearborn, 2001), and personality (Westneat et al., 2011). However, many studies only measure the amount contributed by each carer within one behavioral dimension of care (e.g., food delivery) and during one stage of offspring development (e.g., provisioning nestlings). We currently know relatively little about how carers contribute across multiple dimensions of care behavior, or how the distribution of contributions impacts outcomes for carers and offspring. Similarly, while many studies have explored how carers change the amount they contribute according to the contributions of others (reviewed in Hatchwell, 1999; Harrison et al., 2009), the fine-scale behavioral rules underpinning carer interactions have only recently attracted serious attention (Johnstone et al., 2014). 
In this mini-review, we discuss how care behavior can be quantified, classifying measurements into three broad groups: how parental care is distributed (i.e., when care occurs along the timeline), the characteristics of care (i.e., variation among different instances of similar care behavior), and the interactions between carers (i.e., whether one carer's behavior is associated with the behavior of others). Our aim is to illustrate the variety of questions that can be explored using datasets on parental care, and some of the statistical and technical considerations that arise when doing so. We review existing studies and analysis methods that have addressed these different aspects of care behavior, and briefly discuss potential future research directions.

\section{DISTRIBUTION OF CARE}

\section{Rate and Variance}

When individuals deliver discrete, relatively brief care events to offspring (e.g., provisioning, defense), care/visit rates or the mean and variance of carer inter-visit intervals (IVIs) are useful metrics to quantify behavior. Most literally, IVIs refer to the periods between an individual leaving a nest or den and its next arrival (Santema et al., 2017), but IVIs are also commonly characterized as the time between consecutive arrivals (e.g., Johnstone et al., 2014); for clarity we refer to this latter case as the inter-arrival interval (IAI). When discrete care events are somewhat longer (e.g., nest maintenance) it can also be informative to characterize the within (or intra-) visit intervals (WVI) of carers. Passive integrated transponder (PIT) tags are increasingly used to collect large amounts of visit data on provisioning behavior, particularly in cooperative systems with many carers (Browning et al., 2012), but are less valuable when care occurs away from fixed locations like a nest.

The distributions of intervals (of all types) can be compared between individuals and contexts to understand variation in care. Intervals are often approximately gamma- or inverse-gamma distributed, as they are bounded at zero and often have a (soft) minimum duration that depends on the type of care delivered. They can hence be defined using two independent parameters, scale and shape, that reflect their rate and skewness/variance (Lejeune et al., 2019). In biparental and cooperative systems, the distribution of IAIs by the entire care group is more likely to predict breeding success than those of individual contributors, as the overall amount and distribution of care is what determines outcomes for offspring. Outlier IVIs and IAIs may represent carers taking breaks from caring (e.g., due to self-foraging or disturbance), and hence are useful for characterizing and partitioning longer sample periods to avoid applying inappropriate analyses. When analyzing samples from longer periods of care, intervals (and hence analyses based on them) can be biased as the beginning and end of the sample periods are more likely to cut longer intervals; where possible studies should use either naturally bounded periods, or ensure their sample contains many events and acknowledge the bias (Baldan et al., 2019).

For care delivered over substantial periods (e.g., incubation, babysitting) the proportion of active time carers spend on care, or the proportion of opportunities during which care occurs, are more suitable metrics than the intervals between care events. A typical way to model effects on proportional care is a logistic (Bambini et al., 2018) or binomial (Clutton-Brock et al., 2000) regression when the proportion is derived from counts, or a beta/Dirichlet regression when it is based on continuous numbers (Douma and Weedon, 2019).

\section{Trends}

The rates at which individuals deliver care may vary across a sample period, driven by environmental variation (e.g., weather) or the states of parents or offspring (e.g., hunger). Such variation will affect parental care over the same period, and can limit the usefulness of randomizations used to infer interactions between carers (Baldan et al., 2019). To quantify trends, one simple metric is how strongly intervals are ordered in time (Schlicht et al., 2016), which will identify a linear increase or decrease in rate. A more detailed picture can be obtained by explicitly fitting a model of interval length, with linear and higher-order time terms as predictors. For more complex trends, especially those with periodicity, one could investigate temporal patterns of care using methods developed for time series analysis that have been previously applied to other aspects of behavior, such as cross-correlations (Hall et al., 2014) or wavelet analysis (Zhang et al., 2017). An alternative approach is to group care events by hour or by day and then fit Poisson-based mixed models to the counts of care behavior, with environmental metrics as covariates (Nomano et al., submitted); the best approach will depend on the study system and length of time analyzed.

\section{Repeatability}

In addition to measuring care variation and trends within an observation period, one can also evaluate whether carer behavior is individually repeatable between observations, and hence infer whether that behavior can be regarded as an individual trait. Repeatability is typically defined as the proportion of variance attributable to the differences among groups of observations, before or after controlling for the effect of confounding factors on the response variable ("adjusted repeatability" in the latter case). Detailed guidance on how to measure and interpret repeatability is beyond the scope of this review; see Nakagawa and Schielzeth (2010) for extensive discussion, and the R package "rptR" for useful analysis methods (Stoffel et al., 2017). While there have been several studies on the repeatability of provisioning (Potti et al., 1999; Nakagawa et al., 2007) and other care behaviors including babysitting (English et al., 2010; Sanderson et al., 2015) there is substantial scope for further study.

\section{Multi-Stage Investment}

Empirical studies often focus on a single stage of parental care, or assume each stage is broadly independent. However, theory suggests that investment during earlier stages (e.g., egg-laying) can influence investment rules in later stages (e.g., provisioning), particularly if the breeding female can control the number (Smith and Härdling, 2000; Savage et al., 2013) or quality (Savage et al., 2015) of offspring. These predictions are supported by empirical studies (Russell et al., 2007; Canestrari et al., 2011), but our understanding of multi-stage investment dynamics 
is still incomplete, particularly how these are influenced by environmental conditions (Langmore et al., 2016). As with any form of adaptive plasticity, for between-stage strategies to evolve the environment must be both variable and predictable on the timescale of the care periods, a concept familiar from research on transgenerational effects (Proulx and Teotónio, 2017).

\section{CHARACTERISTICS OF CARE}

\section{Variation Among Events}

Not all instances of a particular care behavior are equivalent from the perspectives of either parents or offspring. For example, provisioned food can vary in mass or nutritional content, and hence carers can vary in contributions without differences in provisioning rate. The size of prey delivered can vary with carer sex (Colombelli-Négrel and Kleindorfer, 2010), these differences may vary with offspring age (Wiebe and Slagsvold, 2009), and males and females may differ in the prey type delivered to offspring (Fraser et al., 2006). Similar considerations apply to other forms of parental care; for example mobbing behavior can vary not only in its frequency but also in the intensity of each event (e.g., contact vs. non-contact) (Strnad et al., 2012), and incubation may be costly to younger but not older carers (Heinsohn and Cockburn, 1994). Characteristics of care events can also affect IVIs, for example larger food items being brought to offspring after parents have been away for longer (Grieco, 2002), altering inferences about carer investment.

\section{Favoritism}

When parents deliver care to multiple offspring, the amount each offspring receives is important for the overall outcome of the breeding attempt. Certain offspring may be more dominant or beg more intensively (Drummond, 2006), or carers may preferentially feed some offspring over others due to expected returns (Jeon, 2008) or favor different offspring if their costs or benefits differ (Lessells, 2002). For example, male offspring may receive more food if mothers preferentially provision sons over daughters (Mainwaring et al., 2011), and parents may adjust which offspring they provision based on offspring age and perceived quality (Avilés et al., 2011).

One metric to characterize this variation is a "skew index" (Pamilo and Crozier, 1996; Shen et al., 2010), which varies from 0 (complete equality) to 1 (one offspring receives all the food). When care to individual offspring can be quantified precisely, for example using video cameras deployed inside nests, comparing between offspring any of the above metrics for the distribution of care can also reveal differences in carer delivery behavior.

\section{Non-care and Deception}

Carers sometimes visit offspring without providing care. For example, individuals bringing food to dependent offspring may consume it themselves in so-called "false feeding" behavior, perhaps representing a deceptive strategy to lower the costs of care (Boland et al., 1997), or a non-deceptive mediation of carer need against offspring need (Canestrari et al., 2010). Identifying deception is non-trivial, as apparent false-feeding can occur when carers visit offspring that are fully satiated; approaches to identify deception include using remote video monitoring to score offspring begging behavior or attempts to feed offspring (Young et al., 2013), measuring latency between arrival and selfconsumption (or departure with food item) compared to normal visits, and testing whether false-feeds are less likely to occur when the provisioner is observed (Boland et al., 1997; Young et al., 2013). In some species such as the bell miner (Manorina melanophrys) carers may also only partially deliver food items; whether these events are treated as false-feeds or not should depend whether they can be more parsimoniously explained by (for example) difficulties in prey transfer (McDonald et al., 2007).

Visits to offspring in which carers arrive without food (and provide no other care) could also be classified as false-feeding, but might instead represent carers updating information about offspring hunger. When carers cannot easily monitor each other's contributions, and visiting offspring is much less costly than finding and delivering food, this additional information is especially valuable to correctly distribute care.

\section{INTERACTIONS DURING CARE}

\section{Negotiation}

As the benefits of care are shared but the costs personal, carers have a conflict of interest over how much each contributes, and this should affect their investment decisions (Trivers, 1972). Theory suggests that individuals in biparental species should respond to changes in the contributions of others by incompletely compensating, both over evolutionary (Houston and Davies, 1985) and behavioral (McNamara et al., 1999) timescales. Further models suggest that incomplete compensation should also occur in cooperative systems (Johnstone, 2011), and that high responsiveness (McNamara et al., 2003), asymmetric information (Johnstone and Hinde, 2006) or threshold effects (Jones et al., 2002) can modify predictions. Empirical work on biparental species largely supports incomplete compensation as the usual response to changes in partner investment, albeit with substantial variation (Harrison et al., 2009) and often sex differences (e.g., Iserbyt et al., 2015). However, cooperative species adopt more diverse investment rules (Hatchwell, 1999), including responding to both the composition and size of the care group (Brouwer et al., 2014) potentially due to greater variation in care during later stages (Savage et al., 2013).

Testing theoretical predictions about negotiation requires careful experiments to manipulate offspring demand (actual or perceived) or carer costs, and monitoring parental responses. The mechanisms through which individuals negotiate are still poorly understood; vocal communication is likely to play a major role (Bell et al., 2010; Boucaud et al., 2016), and as negotiations could also be mediated indirectly through offspring need (Lessells and McNamara, 2012) negotiation behavior is likely to be highly system-specific.

\section{Alternation}

Theoretical work suggests that "turn-taking" - carers alternating contributions-can resolve sexual conflict efficiently (Johnstone et al., 2014), and provided the costs and benefits of care are time-dependent this does not require individuals to monitor 
each other perfectly (Johnstone and Savage, 2019). Empirical work has suggested that several species indeed alternate more than expected by chance, however questions remain over the mechanism of interaction and how strongly this turn-taking is driven by environmental variation vs. individual responsiveness (Ihle et al., 2019).

Turn-taking can be quantified using the proportion of alternated visits (Bebbington and Hatchwell, 2016; Iserbyt et al., 2017) or the log-odds of the deviation between observed and expected number of alternated visits (Baldan et al., in press). Alternatively, the runs test (Wald and Wolfowitz, 1940) can investigate whether two carers alternate more than expected (Johnstone et al., 2014), and a modified version of the test is also applicable to cooperative species (Sheskin, 2011; Khwaja et al., 2017).

Incorporating time information as well as visit sequences, continuous-time Markov models can be used to investigate patterns of alternation in biparental (Johnstone et al., 2014) and cooperative (Savage et al., 2017) species. These analyses can be applied in R using packages such as "msm" (Jackson, 2011), or more simply calculated directly from visit times if withinobservation covariates are not required (Savage et al., in review). If enough data exist to characterize the distribution of care intervals precisely, an alternative approach is to fit a semi-Markov model explicitly using the relevant distribution. Such models can be implemented using (e.g.,) the "SemiMarkov" package in $\mathrm{R}$ (Król and Saint-Pierre, 2015), although to our knowledge this method has yet to be applied to care behavior.

\section{Synchrony}

Depending on the system and behavior in question, pairs or groups synchronizing care activities might have either a positive or negative impact on the success of a breeding attempt. Visiting offspring can increase predation risk (Martin et al., 2000), leading to groups that synchronize visits having increased breeding success (Raihani et al., 2010). Similarly, synchronizing visits might reduce sibling competition by providing resources to more offspring simultaneously (Shen et al., 2010). In contrast, if visits do not increase predation risk, carers deliver multiple (or divisible) food items, and offspring satiate quickly, then carers should deliberately separate their care contributions (antisynchrony). Beyond the impacts on offspring, individuals might also benefit from synchronizing their visits to advertise their contributions to or monitor other group members (Doutrelant and Covas, 2007), particularly in a "pay-to-stay" cooperative system (Gaston, 1978; Kokko et al., 2002).

One method to quantify synchrony is to characterize a particular visit as synchronous when another individual also provides care within a certain window (Mariette and Griffith, 2012) and then use the square root arcsine-transformed proportion of synchronous care events as a measure of overall synchrony (Mariette and Griffith, 2015). This is appropriate in systems with relatively low care rates and brief care events, but can be sensitive to the window chosen. Alternatively, for more frequent or longer care behaviors one can cross-correlate the time series of care contributions by each individual (Savage et al., 2017), and for both methods randomizations can be used to generate expected levels of synchrony. Potentially useful analysis methods have also been developed in neurobiology, where quantifying the relationships between a number of neural spike trains is a common problem (Oram et al., 2001; Shimazaki et al., 2012), however these have yet to be applied to care behavior.

Alternation and synchrony together provide a good picture of individual interactions (Koenig and Walters, 2016), and investigating both is also important because the interpretation of each metric depends partly on the other. For example, a strict pattern of alternation may suggest that individuals are adopting a turn-taking rule under low synchrony, but under high synchrony an alternative explanation would be that individuals forage together and individual differences (e.g., from state or personality) result in one consistently visiting before the other.

\section{Task Specialization}

In many species care occurs simultaneously across multiple behavioral dimensions. For example, in an altricial bird carers may need to feed and brood offspring, maintain the nest, remove fecal sacs, and mob nest predators. Pairs and groups of carers may be comprised of individuals that specialize in particular behaviors and/or generalist individuals, driven by differences in the costs and benefits of each behavior (Arnold et al., 2005). The degree of specialization can change over time (Iserbyt et al., 2017), and within activities carers may sub-specialize (e.g., by food type) or partition roles in time (e.g., helpers rearing first broods while breeders re-nest; Ridley and Raihani, 2008). Comparing parental investment across modalities can be challenging as costs are often accrued in a different "currency" for different care activities and contexts (e.g., mortality via predation risk when mobbing vs. condition via lost self-foraging time during provisioning), but such comparisons are important as these behaviors trade-off against each other (Mutzel et al., 2013).

To investigate factors influencing (e.g.,) the type of prey being delivered to offspring, one approach is to fit the proportional abundance of each prey type as response terms in a (mixed-effect) multinomial logistic regression, to avoid conflating variation in the proportion of each prey type with that of the others (Browning et al., 2012). These models can be most precisely fitted using Markov chain Monte Carlo (MCMC) Bayesian methods, for example with the R packages "RStan" and "MCMCglmm" (Hadfield, 2010; Stan Development Team, 2018). Correlations among the random effects of such models are potentially informative for elucidating individual trade-offs among care behaviors; for discussion of these effects and a detailed treatment of methods around the multinomial analysis of behavior see Koster and McElreath (2017).

\section{DISCUSSION}

In this mini-review we have illustrated that how care is distributed, how care events vary, and how carers interact, each have important consequences for carers and offspring. Our review also illuminates a number of questions that remain despite the vast literature on parental care. Firstly, aside from visit rates, we still know relatively little about how patterns of care are influenced by the ecological (predation, food 
distribution, etc.), environmental (temperature, weather, etc.), physiological (hormone, individual condition), and behavioral (foraging paradigm, pair stability, etc.) contexts of care. Secondly, while provisioning has been well-studied, other dimensions and stages of care-and how these influence each other-require far more attention. Both theory and empirical work suggests these can strongly impact carer behavior to the point that simply measuring one stage and dimension is insufficient.

We advocate both for more in-depth analyses of care behavior, and for raw parental care data from existing studies to be deposited alongside relevant publications, published as data papers, and shared with those interested in applying further analyses where feasible. Collectively, unpublished care data has the potential to greatly advance our understanding of how individuals provide for their offspring.

Our review is restricted to the quantification of carer behavior, but this is inextricably linked to the overall care paradigm, to the behavior of offspring, and to environmental variation. Many species exhibit two or more of the five main patterns of care (none, mother only, father only, biparental, cooperative), often within the same population (Persson and Öhrström, 1989; Webb et al., 1999). Additionally, offspring vary across species in their ability to influence care delivery, with consequences for

\section{REFERENCES}

Arnold, K. E., Owens, I. P. F., and Goldizen, A. W. (2005). Division of labour within cooperatively breeding groups. Behaviour 142, 1577-1590. doi: 10.1163/156853905774831927

Avilés, J. M., Parejo, D., and Rodríguez, J. (2011). Parental favouritism strategies in the asynchronously hatching European Roller (Coracias garrulus). Behav. Ecol. Sociobiol. 65, 1549-1557. doi: 10.1007/s00265-011-1164-8

Baldan, D., Curk, T., Hinde, C. A., and Lessells, C. M. (2019). Alternation of nest visits varies with experimentally manipulated workload in brood-provisioning great tits. Anim. Behav. 156, 139-146. doi: 10.1016/j.anbehav.2019.08.004

Baldan, D., Hinde, C. A., and Lessells, C. M. (in press). Turn-taking between provisioning parents: partitioning alternation. Front. Ecol. Evol.

Bambini, G., Schlicht, E., and Kempenaers, B. (2018). Patterns of female nest attendance and male feeding throughout the incubation period in Blue Tits Cyanistes caeruleus. IBIS 161, 50-65. doi: 10.1111/ibi.12614

Bebbington, K., and Hatchwell, B. J. (2016). Coordinated parental provisioning is related to feeding rate and reproductive success in a songbird. Behav. Ecol. 27, 652-659. doi: 10.1093/beheco/arv198

Bell, M. B. V., Radford, A. N., Smith, R. A., Thompson, A. M., and Ridley, A. R. (2010). Bargaining babblers: vocal negotiation of cooperative behaviour in a social bird. Proc. R. Soc. B Biol. Sci. 277, 3223-3228. doi: 10.1098/rspb.2010.0643

Boland, C. R. J., Heinsohn, R., and Cockburn, A. (1997). Deception by helpers in cooperatively breeding white-winged chougts and its experimental manipulation. Behav. Ecol. Sociobiol. 41, 251-256. doi: 10.1007/s002650050386

Boucaud, I. C. A., Mariette, M. M., Villain, A. S., and Vignal, C. (2016). Vocal negotiation over parental care? Acoustic communication at the nest predicts partners' incubation share. Biol. J. Linn. Soc. 117, 322-336. doi: $10.1111 /$ bij.12705

Brouwer, L., van de Pol, M., and Cockburn, A. (2014). The role of social environment on parental care: offspring benefit more from the presence of female than male helpers. J. Anim. Ecol. 83, 491-503. doi: 10.1111/1365-2656.12143

Browning, L. E., Young, C. M., Savage, J. L., Russell, D. J. F., Barclay, H., Griffith, S. C., et al. (2012). Carer provisioning rules in an obligate cooperative breeder: prey type, size and delivery rate. Behav. Ecol. Sociobiol. 66, 1639-1649. doi: 10.1007/s00265-012-1419-Z investment levels, pre-natal effects and parent-offspring conflict (Hinde et al., 2010). Furthermore, most studies are time- and location-restricted, limiting our understanding of the effects of environmental variation. Integrating these complexities with a more detailed picture of carer behavior remains a key challenge for behavioral ecology.

\section{AUTHOR CONTRIBUTIONS}

JS and $\mathrm{CH}$ : conceptualization, writing, review, and editing. JS: writing-original draft.

\section{FUNDING}

JS was supported by funding from the Natural Environment Research Council.

\section{ACKNOWLEDGMENTS}

We thank Kate Lessells, Rufus Johnstone, Fumiaki Nomano, Wendt Müller, Arne Iserbyt, and Jodie Crane for helpful discussion.

Canestrari, D., Marcos, J. M., and Baglione, V. (2011). Helpers at the nest compensate for reduced maternal investment in egg size in carrion crows. J. Evol. Biol. 24, 1870-1878. doi: 10.1111/j.1420-9101.2011.0 2313.x

Canestrari, D., Vera, R., Chiarati, E., Marcos, J. M., Vila, M., and Baglione, V. (2010). False feeding: the trade-off between chick hunger and caregivers needs in cooperative crows. Behav. Ecol. 21, 233-241. doi: 10.1093/beheco/arp177

Clutton-Brock, T. H., Brotherton, P. N. M., O’Riain, M. J., Griffin, A. S., Gaynor, D., Sharpe, L. L., et al. (2000). Individual contributions to babysitting in a cooperative mongoose, Suricata suricatta. Proc. R. Soc. B Biol. Sci. 267, 301-305. doi: $10.1098 / \mathrm{rspb} .2000 .1000$

Colombelli-Négrel, D., and Kleindorfer, S. (2010). Video nest monitoring reveals male coloration-dependant nest predation and sex differences in prey size delivery in a bird under high sexual selection. J. Ornithol. 151, 507-512. doi: 10.1007/s10336-009-0480-5

Dearborn, D. C. (2001). Body condition and retaliation in the parental effort decisions of incubating great frigatebirds (Fregata minor). Behav. Ecol. 12, 200-206. doi: 10.1093/beheco/12.2.200

Douma, J. C., and Weedon, J. T. (2019). Analysing continuous proportions in ecology and evolution: a practical introduction to beta and Dirichlet regression. Methods Ecol. Evol. 2019, 1412-1430. doi: 10.1111/2041-210 X.13234

Doutrelant, C., and Covas, R. (2007). Helping has signalling characteristics in a cooperatively breeding bird. Anim. Behav. 74, 739-747. doi: 10.1016/j.anbehav.2006.11.033

Drummond, H. (2006). Dominance in vertebrate broods and litters. Q. Rev. Biol. 81, 3-32. doi: 10.1086/503922

English, S., Nakagawa, S., and Clutton-Brock, T. H. (2010). Consistent individual differences in cooperative behaviour in meerkats (Suricata suricatta). J. Evol. Biol. 23, 1597-1604. doi: 10.1111/j.1420-9101.2010. 02025.x

Fraser, G. S., Jones, I. L., and Hunter, F. M. (2006). Male-female differences in parental care in monogamous crested auklets. Condor 104, 413. doi: 10.1650/ 0010-5422(2002)104[0413:MFDIPC]2.0.CO;2

Gaston, A. J. (1978). The evolution of group territorial behavior and cooperative breeding. Am. Nat. 112, 1091-1100. doi: 10.1086/2 83348 
Grieco, F. (2002). Time constraint on food choice in provisioning blue tits, Parus caeruleus: the relationship between feeding rate and prey size. Anim. Behav. 64, 517-526. doi: 10.1006/anbe.2002.3073

Hadfield, J. D. (2010). MCMC methods for multi-response generalized linear mixed models: the MCMCglmm R Package. J. Stat. Softw. 33, 1-22. Available online at: https://www.jstatsoft.org/article/view/v033i02

Hall, K., Oram, M. W., Campbell, M. W., Eppley, T. M., Byrne, R. W., and De Waal, F. B. M. (2014). Using cross correlations to investigate how chimpanzees (Pan troglodytes) use conspecific gaze cues to extract and exploit information in a foraging competition. Am. J. Primatol. 76, 932-941. doi: 10.1002/ajp.22279

Harrison, F., Barta, Z., Cuthill, I. C., and Székely, T. (2009). How is sexual conflict over parental care resolved? A meta-analysis. J. Evol. Biol. 22, 1800-1812. doi: 10.1111/j.1420-9101.2009.01792.x

Hatchwell, B. J. (1999). Investment strategies of breeders in avian cooperative breeding systems. Am. Nat. 154, 205-219. doi: 10.1086/303227

Heinsohn, R. G., and Cockburn, A. (1994). Helping is costly to young birds in cooperatively breeding white-winged choughs. Proc. R. Soc. B Biol. Sci. 256, 293-298. doi: 10.1098/rspb.1994.0083

Hinde, C. A., Johnstone, R. A., and Kilner, R. M. (2010). Parent-offspring conflict and coadaptation. Science 327, 1373-1376. doi: 10.1126/science.1186056

Houston, A. I., and Davies, N. B. (1985). "The evolution of cooperation and life history in the dunnock, Prunella modularis," in Behavioural Ecology: Ecological Consequences of Adaptive Behaviour, eds R. M. Sibly and R. H. Smith (Oxford: Blackwell Scientific Publications), 471-487.

Ihle, M., Pick, J. L., Winney, I. S., Nakagawa, S., and Burke, T. (2019). Measuring up to reality: null models and analysis simulations to study parental coordination over provisioning offspring. Front. Ecol. Evol. 7:142. doi: 10.3389/fevo.2019.00142

Iserbyt, A., Farrell, S., Eens, M., and Müller, W. (2015). Sex-specific negotiation rules in a costly conflict over parental care. Anim. Behav. 100, 52-58. doi: 10.1016/j.anbehav.2014.11.014

Iserbyt, A., Fresneau, N., Kortenhoff, T., Eens, M., and Müller, W. (2017). Decreasing parental task specialization promotes conditional cooperation. Sci. Rep. 7:6565. doi: 10.1038/s41598-017-06667-1

Jackson, C. (2011). Multi-state models for panel data: the msm package for R. J. Stat. Softw. 38, 1-29. doi: 10.18637/jss.v038.i08

Jeon, J. (2008). Evolution of parental favoritism among different-aged offspring. Behav. Ecol. 19, 344-352. doi: 10.1093/beheco/arm136

Johnstone, R. A. (2011). Load lightening and negotiation over offspring care in cooperative breeders. Behav. Ecol. 22, 436-444. doi: 10.1093/beheco/arq190

Johnstone, R. A., and Hinde, C. A. (2006). Negotiation over offspring carehow should parents respond to each other's efforts? Behav. Ecol. 17, 818-827. doi: 10.1093/beheco/arl009

Johnstone, R. A., Manica, A., Fayet, A. L., Stoddard, M. C., Rodriguez-Gironés, M. A., and Hinde, C. A. (2014). Reciprocity and conditional cooperation between great tit parents. Behav. Ecol. 25, 216-222. doi: 10.1093/beheco/ art109

Johnstone, R. A., and Savage, J. L. (2019). Conditional cooperation and turntaking in parental care. Front. Ecol. Evol. 7:335. doi: 10.3389/fevo.2019. 00335

Jones, K. M., Ruxton, G. D., and Monaghan, P. (2002). Model parents: is full compensation for reduced partner nest attendance compatible with stable biparental care? Behav. Ecol. 13, 838-843. doi: 10.1093/beheco/13.6.838

Khwaja, N., Preston, S. A. J., Hatchwell, B. J., Briskie, J. V., Winney, I. S., and Savage, J. L. (2017). Flexibility but no turn-taking in provisioning riflemen (Acanthisitta chloris). Anim. Behav. 125, 25-31. doi: 10.1016/j.anbehav.2016.12.021

Koenig, W. D., and Walters, E. L. (2016). Provisioning patterns in the cooperatively breeding acorn woodpecker: does feeding behaviour serve as a signal? Anim. Behav. 119, 125-134. doi: 10.1016/j.anbehav.2016.06.002

Kokko, H., Johnstone, R. A., and Wright, J. (2002). The evolution of parental and alloparental effort in cooperatively breeding groups: when should helpers pay to stay? Behav. Ecol. 13, 291-300. doi: 10.1093/beheco/13.3.291

Koster, J., and McElreath, R. (2017). Multinomial analysis of behavior: statistical methods. Behav. Ecol. Sociobiol. 71, 138. doi: 10.1007/s00265-017-2363-8

Król, A., and Saint-Pierre, P. (2015). SemiMarkov : an R package for parametric estimation in multi-state semi-Markov models. J. Stat. Softw. 66, 1-16. doi: $10.18637 /$ jss.v066.i06
Langmore, N. E., Bailey, L. D., Heinsohn, R. G., Russell, A. F., and Kilner, R. M. (2016). Egg size investment in superb fairy- wrens : helper effects are modulated by climate. Proc. R. Soc. B. 283:20161875. doi: 10.1098/rspb. 2016.1875

Lejeune, L. A., Savage, J. L., Bründl, A. C., Thiney, A. C., Chaine, A. S., and Russell, A. F. (2019). Environmental effects on parental care visitation patterns in blue tits Cyanistes caeruleus. Front. Ecol. Evol. 7:356. doi: 10.3389/fevo.2019. 00356

Lessells, C. M. (2002). Parentally biased favouritism: why should parents specialize in caring for different offspring? Philos. Trans. R. Soc. B Biol. Sci. 357, 381-403. doi: 10.1098/rstb.2001.0928

Lessells, C. M., and McNamara, J. M. (2012). Sexual conflict over parental investment in repeated bouts: negotiation reduces overall care. Proc. R. Soc. B Biol. Sci. 279, 1506-1514. doi: 10.1098/rspb. 2011.1690

Liker, A., Freckleton, R. P., Remeš, V., and Székely, T. (2015). Sex differences in parental care: gametic investment, sexual selection, and social environment. Evolution. 69, 2862-2875. doi: 10.1111/evo.12786

Mainwaring, M. C., Lucy, D., and Hartley, I. R. (2011). Parentally biased favouritism in relation to offspring sex in zebra finches. Behav. Ecol. Sociobiol. 65, 2261-2268. doi: 10.1007/s00265-011-1235-x

Mariette, M. M., and Griffith, S. C. (2012). Nest visit synchrony is high and correlates with reproductive success in the wild Zebra finch Taeniopygia guttata. J. Avian Biol. 43, 131-140. doi: 10.1111/j.1600-048X.2012.0 5555.x

Mariette, M. M., and Griffith, S. C. (2015). The adaptive significance of provisioning and foraging coordination between breeding partners. Am. Nat. 185, 270-280. doi: 10.1086/679441

Martin, T. E., Scott, J., and Menge, C. (2000). Nest predation increases with parental activity: separating nest site and parental activity effects. Proc. R. Soc. B Biol. Sci. 267, 2287-2293. doi: 10.1098/rspb.2000.1281

McDonald, P. G., Kazem, A. J. N., and Wright, J. (2007). A critical analysis of "false-feeding" behavior in a cooperatively breeding bird: disturbance effects, satiated nestlings or deception? Behav. Ecol. Sociobiol. 61, 1623-1635. doi: 10.1007/s00265-007-0394-2

McNamara, J. M., Gasson, C. E., and Houston, A. I. (1999). Incorporating rules for responding into evolutionary games. Nature 401, 368-371. doi: 10.1038/ 43869

McNamara, J. M., Houston, A. I., Barta, Z., and Osorno, J. L. (2003). Should young ever be better off with one parent than with two? Behav. Ecol. 14, 301-310. doi: 10.1093/beheco/14.3.301

Mutzel, A., Blom, M. P. K., Spagopoulou, F., Wright, J., Dingemanse, N. J., and Kempenaers, B. (2013). Temporal trade-offs between nestling provisioning and defence against nest predators in blue tits. Anim. Behav. 85, 1459-1469. doi: 10.1016/j.anbehav.2013.03.043

Nakagawa, S., Gillespie, D. O. S., Hatchwell, B. J., and Burke, T. (2007) Predictable males and unpredictable females: sex difference in repeatability of parental care in a wild bird population. J. Evol. Biol. 20, 1674-1681. doi: 10.1111/j.1420-9101.2007.01403.x

Nakagawa, S., and Schielzeth, H. (2010). Repeatability for Gaussian and nonGaussian data: a practical guide for biologists. Biol. Rev. 85, 935-956. doi: 10.1111/j.1469-185X.2010.00141.x

Oram, M. W., Hatsopoulos, N. G., Richmond, B. J., and Donoghue, J. P. (2001). Excess synchrony in motor cortical neurons provides redundant direction information with that from coarse temporal measures. J. Neurophysiol. 86, 1700-1716. doi: 10.1152/jn.2001.86.4.1700

Ortega, S., Sánchez-Macouzet, O., Urrutia, A., Rodríguez, C., and Drummond, H. (2017). Age-related parental care in a long-lived bird: implications for offspring development. Behav. Ecol. Sociobiol. 71:132. doi: 10.1007/s00265-0172364-7

Pamilo, P., and Crozier, R. H. (1996). Reproductive skew simplified. Oikos 75, 533-535. doi: 10.2307/3545895

Persson, O., and Öhrström, P. (1989). A new avian mating system : ambisexual polygamy in the penduline tit Remiz pendulinus. Scand. J. Ornithol. 20, 105-111. doi: $10.2307 / 3676876$

Potti, J., Moreno, J., and Merino, S. (1999). Repeatability of parental effort in male and female Pied Flycatchers as measured with double labeled water. Can. J. Zool. 77, 174-179. doi: 10.1139/z98-196 
Proulx, S. R., and Teotónio, H. (2017). What kind of maternal effects can be selected for in fluctuating environments? Am. Nat. 189, E118-E137. doi: $10.1086 / 691423$

Raihani, N. J., Nelson-Flower, M. J., Moyes, K., Browning, L. E., and Ridley, A. R. (2010). Synchronous provisioning increases brood survival in cooperatively breeding pied babblers. J. Anim. Ecol. 79, 44-52. doi: 10.1111/j.1365-2656.2009.01606.x

Ridley, A. R., and Raihani, N. J. (2008). Task partitioning increases reproductive output in a cooperative bird. Behav. Ecol. 19, 1136-1142. doi: 10.1093/beheco/arn097

Russell, A. F., Langmore, N. E., Cockburn, A., Astheimer, L. B., and Kilner, R. M. (2007). Reduced egg investment can conceal helper effects in cooperatively breeding birds. Science 317, 941-944. doi: 10.1126/science.1146037

Saether, B.-E. (1994). Food provisioning in relation to reproductive strategy in altricial birds: a comparison of two hypotheses. Evolution 48, 1397-1406. doi: 10.1111/j.1558-5646.1994.tb05324.x

Sanderson, J. L., Stott, I., Young, A. J., Vitikainen, E. I. K., Hodge, S. J., and Cant, M. A. (2015). The origins of consistent individual differences in cooperation in wild banded mongooses, Mungos mungo. Anim. Behav. 107, 193-200. doi: 10.1016/j.anbehav.2015.06.022

Santema, P., Schlicht, E., Schlicht, L., and Kempenaers, B. (2017). Blue tits do not return faster to the nest in response to either short- or long-term begging playbacks. Anim. Behav. 123, 117-127. doi: 10.1016/j.anbehav.2016.10.016

Savage, J. L., Browning, L. E., Manica, A., Russell, A. F., and Johnstone, R. A. (2017). Turn-taking in cooperative offspring provisioning: by-product of individual provisioning behaviour or active response rule? Behav. Ecol. Sociobiol. 71:162. doi: 10.1007/s00265-017-2391-4

Savage, J. L., Russell, A. F., and Johnstone, R. A. (2013). Maternal costs in offspring production affect investment rules in joint rearing. Behav. Ecol. 24, 750-758. doi: 10.1093/beheco/ars203

Savage, J. L., Russell, A. F., and Johnstone, R. A. (2015). Maternal allocation in cooperative breeders: should mothers match or compensate for expected helper contributions? Anim. Behav. 102, 189-197. doi: 10.1016/j.anbehav.201 5.01 .015

Schlicht, E., Santema, P., Schlicht, R., and Kempenaers, B. (2016). Evidence for conditional cooperation in biparental care systems? A comment on Johnstone et al. Behav. Ecol. 27, e2-e5. doi: 10.1093/beheco/ arw036

Shen, S.-F., Chen, H.-C., Vehrencamp, S. L., and Yuan, H.-W. (2010). Group provisioning limits sharing conflict among nestlings in joint-nesting Taiwan yuhinas. Biol. Lett. 6, 318-321. doi: 10.1098/rsbl.2009.0909

Sheskin, D. J. (2011). Handbook of Parametric and Nonparametric Statistical Procedures, 5th Edn. Boca Raton, FL: CRC Press.

Shimazaki, H., Amari, S., ichi, Brown, E. N., and Grün, S. (2012). Statespace analysis of time-varying higher-order spike correlation for multiple neural spike train data. PLoS Comput. Biol. 8:e1002385. doi: 10.1371/journal.pcbi.1002385

Smith, H. G., and Härdling, R. (2000). Clutch size evolution under sexual conflict enhances the stability of mating systems. Proc. R. Soc. B Biol. Sci. 267, 2163-2170. doi: 10.1098/rspb.2000.1264

Stan Development Team (2018). RStan: The R interface to Stan.

Stoffel, M. A., Nakagawa, S., and Schielzeth, H. (2017). rptR: repeatability estimation and variance decomposition by generalized linear mixed-effects models. Methods Ecol. Evol. 8, 1639-1644. doi: 10.1111/2041-210X.12797

Strnad, M., Němec, M., Vesel,ý, P., and Fuchs, R. (2012). Red-backed Shrikes (Lanius collurio) adjust the mobbing intensity, but not mobbing frequency, by assessing the potential threat to themselves from different predators. Ornis Fenn. 89, 206-215.

Trivers, R. L. (1972). "Parental investment and sexual selection," in Sexual Selection and the Descent of Man 1871-1971, ed. B. Campbell (Chicago, IL: AldineAtherton), 136-207.

Wald, A., and Wolfowitz, J. (1940). On a test whether two samples are from the same population. Ann. Math. Stat. 11, 147-162. doi: 10.1214/aoms/11777 31909

Webb, J., Houston, A., McNamara, J., and Szekely, T. (1999). Multiple patterns of parental care. Anim. Behav. 58, 983-993. doi: 10.1006/anbe.199 9.1215

Westneat, D. F., Hatch, M. I., Wetzel, D. P., and Ensminger, A. L. (2011). Individual variation in parental care reaction norms: integration of personality and plasticity. Am. Nat. 178, 652-667. doi: 10.1086/662173

Wiebe, K. L., and Slagsvold, T. (2009). Parental sex differences in food allocation to junior brood members as mediated by prey size. Ethology 115, 49-58. doi: 10.1111/j.1439-0310.2008.01580.x

Young, C. M., Browning, L. E., Savage, J. L., Griffith, S. C., and Russell, A. F. (2013). No evidence for deception over allocation to brood care in a cooperative bird. Behav. Ecol. 24, 70-81. doi: 10.1093/beheco/ars137

Zhang, J., Hull, V., Ouyang, Z., He, L., Connor, T., Yang, H., et al. (2017). Modeling activity patterns of wildlife using time-series analysis. Ecol. Evol. 7, 2575-2584. doi: $10.1002 /$ ece 3.2873

Conflict of Interest: The authors declare that the research was conducted in the absence of any commercial or financial relationships that could be construed as a potential conflict of interest.

Copyright (c) 2019 Savage and Hinde. This is an open-access article distributed under the terms of the Creative Commons Attribution License (CC BY). The use, distribution or reproduction in other forums is permitted, provided the original author(s) and the copyright owner(s) are credited and that the original publication in this journal is cited, in accordance with accepted academic practice. No use, distribution or reproduction is permitted which does not comply with these terms. 